DEPÓSITO LEGAL ZU2020000153

Esta publicación científica en formato digital

es continuidad de la revista impresa

ISSN 0041-8811

E-ISSN 2665-0428

Revista

de la

Universidad

del Tunlia

Fundada en 1947

por el Dr. Jesús Emrique Lossada

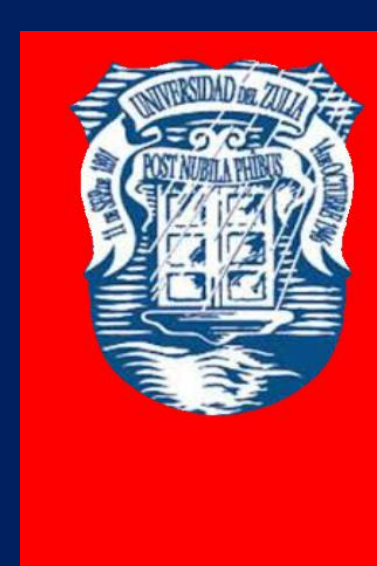

Ciencias

Sociales

y Arte

Aกัต 11 No 31

Septiembre - Diciembre 2021

Tercera ípoca

Maracailbo-Venezuela 


\title{
Calidad de servicio y nivel de satisfacción en el hospital José Cayetano Heredia, Perú
}

\author{
Teolinda Chuquicusma Tocto* \\ Priscila Estelita Luján Vera ** \\ Manuel Jesús Sánchez-Chero *** \\ Yency Miriana Montalban Ramos **** \\ Raquel Silva Juárez $* * * * *$ \\ Carmen Elvira Rosas-Prado ***
}

RESUMEN

La presente investigación tiene como objetivo establecer la relación entre la calidad de servicio y la satisfacción de los usuarios del área de Atención al Asegurado del Hospital José Cayetano Heredia -Perú. La metodología se centra en el enfoque cuantitativo, diseño no experimental, de alcance descriptivo correlacional. La muestra estuvo constituida por 70 usuarios. Se aplicó un cuestionario de 49 items distribuidas en siete dimensiones (tangibilidad, responsabilidad, seguridad, empatía, receptividad, credibilidad y competencia), realizándose el estudio en base al Modelo SERVQUAL. Los resultados encontrados refieren que la calidad de servicio muestra una correlación significativa positiva alta con la satisfacción del usuario en un 0.88 , el nivel de calidad de servicio que brinda el área de atención al asegurado es bajo en un 42.9\%, el nivel de satisfacción de los usuarios del área de atención al asegurado es bajo en un $45.7 \%$. Se concluye que existe una relación significativa entre la calidad de servicio y la satisfacción de atención al usuario.

PALABRAS CLAVE: Calidad de servicio de hospitales, satisfacción del usuario, modelo SERVQUAL

* Docente. Universidad Nacional de Piura. Perú. https://orcid.org/ 0000-0001-6276-3554

**Docente. Universidad Nacional de Frontera. Perú. https://orcid.org/0000-0002-1359-5715

***Docente Investigador. Universidad Nacional de Frontera. Perú. https:/orcid.org/00000003-1646-3037. E-mail: msanchezch@unf.edu.pe

***** Docente. Universidad Nacional de Piura. Perú. https://orcid.org/0000-0003-0817-1819

***** Docente. Universidad Nacional de Frontera. Perú. https://orcid.org/0000-0003-48391533

****** Docente Investigadora. Universidad Señor de Sipán S.A.C. Perú. https://orcid.org/0000-0002-7924-3157 


\section{Quality of service and level of satisfaction in the José Cayetano Heredia hospital, Peru}

ABSTRACT

The objective of this research is to establish the relationship between the quality of service and the satisfaction of the users of the Insured Service area of the José Cayetano Heredia Hospital - Peru. The methodology focuses on the quantitative approach, non-experimental design, with a correlational descriptive scope. The sample consisted of 70 users. A questionnaire of 49 items distributed in seven dimensions (tangibility, responsibility, security, empathy, receptivity, credibility and competence) was applied, carrying out the study based on the SERVQUAL Model. The results found refer that the quality of service shows a high positive significant correlation with user satisfaction in 0.88 , the level of quality of service provided by the area of attention to the insured is low in $42.9 \%$, the level of satisfaction of users in the insured service area is low by $45.7 \%$. It is concluded that there is a significant relationship between service quality and customer service satisfaction.

KEY WORDS: Hospital service quality, user satisfaction, SERVQUAL model

\section{Introducción}

Los sistemas de atención al usuario deben ser para las entidades de salud una de las estrategias de comunicación organizacional más importantes en la prestación de servicios, debido que a través de esta área se mantienen las relaciones interpersonales, donde se evidencia la relevancia de la opinión del usuario respecto al cumplimientos del servicio y el acceso eficaz y eficiente del recurso humano. Los usuarios constituyen el componente vital de las organizaciones; hacia ellos va dirigido el servicio o producto; desde la pequeña y mediana empresa, hasta las corporaciones más grandes (Fariño et al, 2018). Por lo tanto, un producto o servicio ofrecido por una organización posee variados elementos, que parten desde su sistema de administración, reflejando una estructura organizacional que pone en evidencia un estilo de administración y liderazgo enfocado en un sistema y modelo que permite el desarrollo de forma colectiva (Pantoja y Salazar, 2019; Vásquez Gastelumendi et al, 2019), de esta manera se relaciona de forma directa con la satisfacción del usuario convirtiendo la responsabilidad del mismo en universal para todos los elementos de las actividades de la empresa. 
REVISTA DE LA UNIVERSIDAD DEL ZULIA. $3^{a}$ época. Año 11 N³1, 2020

En el Perú el sistema de salud está contemplado como una necesidad básica vital de una sociedad en pleno desarrollo democrático, siendo el derecho de cualquier ciudadano ser atendido de forma inmediata y bajo condiciones de calidad que le permitan sentir seguridad de su salud. El Hospital José Cayetano Heredia de Piura, es una institución pública dedicada a velar por la salud de los ciudadanos de la provincia. Su principal compromiso es mejorar la calidad de vida de las familias, brindándoles servicios de salud y social los cuales están comprendidos dentro del régimen contributivo de la Seguridad Social de Salud, se dedica a velar por el derecho de la población al bienestar y garantizar el libre acceso a prestaciones de salud. Sin embargo, el servicio que ofrece el área de Atención al Asegurado del Hospital Cayetano Heredia a los usuarios se ve afectado por diversos factores como la demora en ser atendidos, lo que genera largas horas de espera y se ve reflejado por las extensas colas, no se realiza reportes de consulta externa oportunamente, no se orienta debidamente al paciente, no se digitan las citas en forma correcta, existe carencia de trabajo en equipo para solucionar problemas del usuario, se denota carencia de empatía en el trato con el paciente y el ambiente no es el adecuado para atender a todos los asegurados.

En consecuencia, se ve afectada la satisfacción del usuario, por lo que es indispensable que se tomen acciones para mejorar la calidad de servicio con el único objetivo de lograr una mejora continua en el trato y la atención de los pacientes que acuden diariamente a solucionar un problema de salud, así como también se sientan cómodos en un ambiente agradable. Por lo expuesto, se planteó como objetivo general establecer la relación entre la calidad de servicio y la satisfacción de los usuarios del área de Atención al Asegurado del Hospital José Cayetano Heredia-Perú.

\section{Calidad}

En los últimos años se ha podido visualizar que la calidad se ha visto condicionada por ciertos factores que han determinado mejoras en los productos ofrecidos en todos los rubros de prestación de servicios públicos y privados, con el único fin de satisfacer las expectativas del mercado nacional e internacional y estar a la vanguardia de las necesidades de la sociedad.

Si damos una mirada desde los inicios de la conceptualización del término "calidad" nos encontramos con (Parasuraman et al, 1985), para quienes la calidad del servicio es el 
REVISTA DE LA UNIVERSIDAD DEL ZULIA. 3época. Año 11 N 31, 2020

resultado de la comparación de las expectativas o deseos del consumidor frente a un proveedor y sus percepciones con respecto al servicio recibido (Lozada y Rodriguez, 2007). De tal forma Deming (1989) sostiene que "la calidad es el grado predecible de uniformidad y fiabilidad a bajo coste y adecuado a las necesidades del mercado". Así mismo, Juran y Gryna (1993) definen la calidad como la adecuación al uso, basándose en el acondicionamiento del diseño del producto o servicio (calidad de diseño) y la medición del grado en que el producto es conforme con dicho diseño (calidad de fabricación o conformidad), cabe resaltar que la calidad de diseño se refiere a las descripciones que debe poseer un producto para dar conformidad a la necesidad de los clientes.

Es así como (Menacho et al, 2020) manifiestan que la calidad tiene relación con la parte psicológica del usuario, debido a que la buena impresión que se pueda percibir delimitará la conceptualización de calidad, desarrollando en las estructuras cognitivas una correcta imagen del servicio a un proceso extendido (Soto et al, 2020).

Este estudio hace utilidad del Modelo SERVQUAL, propuesto por (Parasuraman et al, 2016), cuya finalidad es la evaluación de los servicios; aquí se propone una serie de dimensiones que permiten identificar las falencias en las áreas donde se aplica, cabe indicar que este modelo fue validado por primera vez en Latinoamérica en el año 1992 por Michael Consulting y el Instituto Latinoamericano de Calidad en los Servicios. Es evidente que fue creado por empresas, sin embargo, este modelo es aplicado de manera constante en el sector salud debido a que las dimensiones que lo integran permiten recabar información necesaria para conocimiento en implementación de planes de mejora que consoliden la correcta gestión de los sistemas de salud de Latinoamérica (Castellano et al, 2019). En este sentido, los estudios de calidad y servicio intentan explicar el ámbito competitivo en el que están inmersas las empresas, y las entidades prestadoras de servicio de salud no son ajenas a esta competitividad, las cuales están en constante gestión para mejorar los procesos administrativos que les permita ofrecer una atención mejorada y otorgar un valor adicional a los servicios ofrecidos (Gallardo y Reynaldos, 2014). En la figura 1 se puede observar las dimensiones desarrolladas en este estudio.

2. Calidad de servicio en hospitales 
REVISTA DE LA UNIVERSIDAD DEL ZULIA. 3época. Año $11 \mathrm{~N}^{\circ}$ 31, 2020

La calidad en la prestación de los servicios de salud es un tema de interés para todos los actores sociales que dependen de este proceso, convirtiéndose los médicos, enfermeras, especialistas y todo el personal involucrado de un hospital en los encargados de prestar este servicio (Losada y Rodríguez, 2007).

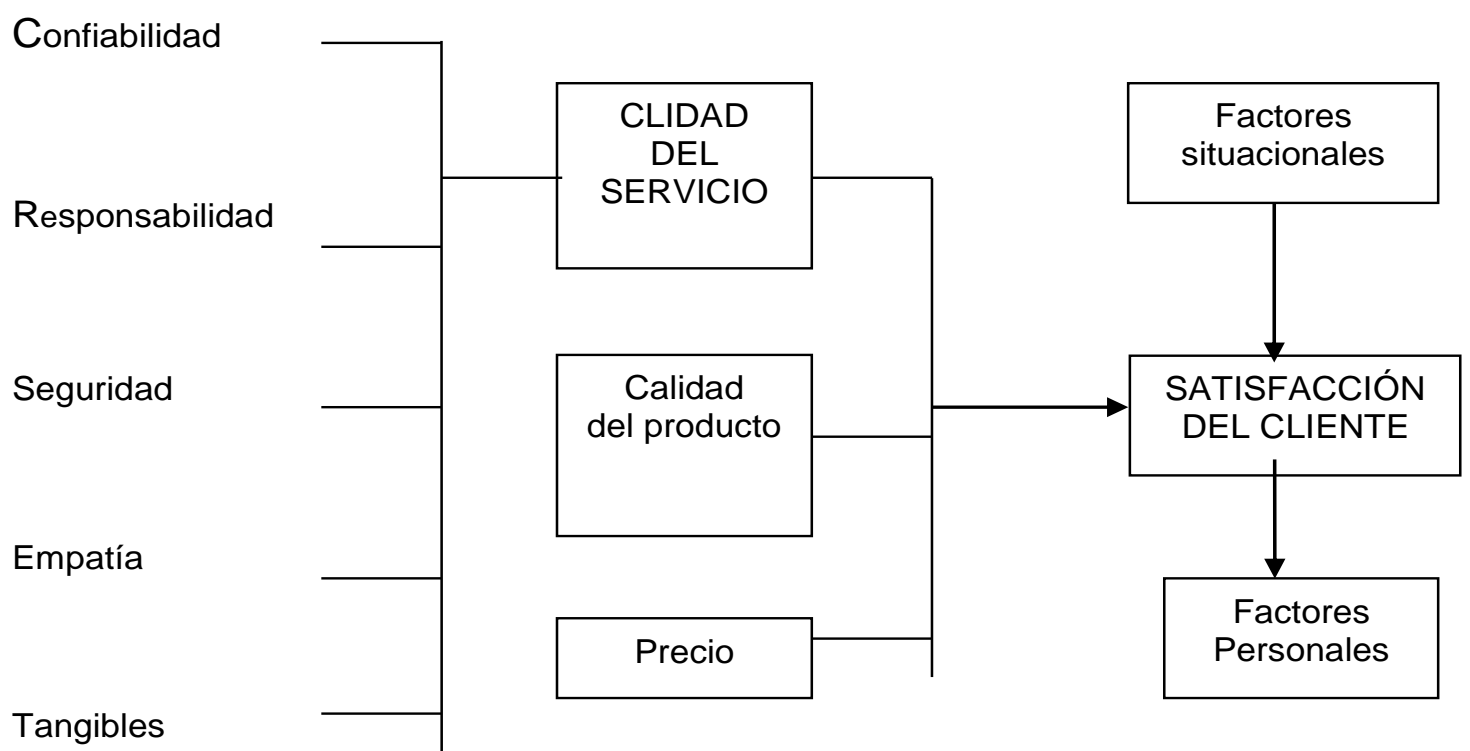

Figura l. Relación de la calidad de servicio con la satisfacción del usuario Fuente: Marketing de Servicios, Zeithmal y Bitner (2002)

Canzio (2019) manifiesta que una atención sanitaria con niveles de estándares alcanzados se identifica por el adecuado desempeño profesional; mientras que Ortiz (2016) sostiene que el resultado de calidad depende del desarrollo interactivo entre la entidad y los usuarios. En este sentido, para (Loli et al, 2014) la calidad de servicio depende de factores socio comunicativo como "la capacidad de respuesta de la organización, la actitud personal, favorable o desfavorable, de sus trabajadores respecto a la atención del cliente y la capacidad profesional de los trabajadores" (Montalvo et al, 2020).

En el Perú, la Ley 26842 Ley General de Salud, en su art.3 menciona que "es el derecho de toda persona a recibir atención médico quirúrgico de emergencia cuando lo solicite, y mientras subsista el estado grave de riesgo para su vida y salud, donde los establecimientos de salud están obligados a prestar la atención sin excepción", asimismo, señala, en su Reglamento General cap. VI, art.83, que "están obligados a prestar atención inmediata a toda 
REVISTA DE LA UNIVERSIDAD DEL ZULIA. 3época. Año $11 \mathrm{~N}^{\circ}$ 31, 2020

persona en situación de emergencia, el médico o médicos que realizan la evaluación correspondiente" (Casaño, 2017). El Ministerio de Salud mediante la presente norma imparte instrucciones para mejorar la calidad de atención que se brinda al paciente en los servicios de emergencia de los establecimientos privados y públicos del sector salud; teniendo en consideración aquella circunstancia súbita o inesperada que requieren atención inmediata al poner en peligro la vida del paciente.

Podesta y Maceda (2018) sostienen que cuando se hace referencia a gestión de la calidad en salud es evidente asociarlo a indicadores como infraestructura (ambientes cómodos y aseados, equipos e implementos de seguridad), material informativo, tecnología, medicina, como también el recurso humano altamente capacitado (Cobo et al, 2018). Sin embargo, la conceptualización transciende los parámetros ya que se tienen que considerar otros aspectos como el desarrollo económico, identidad cultural, gestión administrativa, área legal. Cabe señalar que (Numpaque et al, 2019) coincide al plantear que la mejoría de la calidad percibida se puede conseguir mediante la gestión eficaz y eficiente de los procesos derivados, es decir, que un hospital implementado de equipos tecnológicos, medicina al alcance, infraestructura y optimización del recurso humano para la atención inmediata de pacientes que lo requieran conduciría a la reducción de tiempos de espera, evidenciando un servicio de calidad ante la percepción de los usuarios.

\section{Satisfacción del usuario}

(Cadena et al, 2019) manifiesta que la satisfacción es la posición psicológica que se desarrolla para evolucionar el campo intelectual, lo que causa una sensación de plasencia frente a la consulta clínica y la forma de atención. Para (Numpaque et al, 2019) la satisfacción del usuario respecto a la calidad de los servicios de salud es una temática compleja que se relaciona con diferentes factores como la edad, el nivel educativo, la especialidad quirúrgica por la cual el paciente es atendido y el sistema de seguridad social en salud. Sin embargo (Fernández et al, 2019) la consideran como una respuesta actitudinal y de juicio de valor que el usuario construye producto de su encuentro e interacción con el servicio. Es importante resaltar que el buen trato al usuario genera expectativas significativas, que se internalizan y al mismo tiempo desarrollan una cadena de marketing oral en la comunidad donde el usuario 
REVISTA DE LA UNIVERSIDAD DEL ZULIA. 3época. Año $11 \mathrm{~N}^{\circ}$ 31, 2020

se desenvuelve, produciendo una imagen sostenible de la empresa, respecto al servicio que ofrece.

\section{Metodología}

La investigación se desarrolló bajo el diseño no experimental, de tipo descriptivocorrelacional, donde se realizó la descripción de las variables a estudiar, identificando sus características y comportamientos (Hernández et al, 2010), de tal forma que se determinó si existe relación entre la calidad de servicio y satisfacción del usuario.

El esquema de este diseño fue:

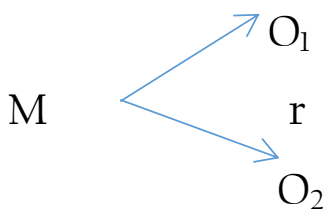

Donde:

$M=$ Es la muestra

$\mathrm{O}_{1}=$ Es la observación a la variable independiente.

$r=$ Es el coeficiente de correlación entre las 02 variables

$\mathrm{O}_{2}=$ Es la observación a la variable dependiente.

La población de estudio estuvo constituida por los 70 usuarios que son atendidos diariamente en el área de atención al asegurado del Hospital José Cayetano Heredia; para ello se escogió durante una semana a 10 pacientes cada día de manera aleatoria, donde se encuestaron a personas de diferentes zonas. La técnica de recolección de datos fue la encuesta, ya que se deseó a partir de ella, obtener la información pertinente y necesaria de la muestra representativa en el estudio. Se utilizó como instrumento para la medición de las variables de estudio el cuestionario de calidad de servicio, el cual estuvo compuesta por 49 ítems, basado en el modelo de SERVQUAL y el cuestionario de satisfacción del usuario. El procesamiento de datos se realizó en el SPSS 21.00. Para evaluar la significancia de este indicador, se tuvo en cuenta que el nivel de significación (Sig.) proporcionado por el programa fuera menor a 0.05 (equivalente a un nivel de significación del 5\%). Para la correlación entre calidad de servicio y satisfacción del usuario, se analizó a través del coeficiente de correlación de Spearman. 
REVISTA DE LA UNIVERSIDAD DEL ZULIA. 3época. Año $11 \mathrm{~N}^{\circ}$ 31, 2020

\section{Resultados}

En La tabla l se evidencia que la calidad de servicio que se brinda en el área de atención al asegurado del Hospital José Cayetano Heredia demuestra un promedio de 2.21 puntos, considerándose como bajo. Así mismo, los promedios en cada uno de las dimensiones que la componen (tangibilidad, seguridad, responsabilidad y empatía). Los promedios ligeramente muestran puntos entre 2.19 y 2.23, reflejándose como niveles bajos en todos sus componentes. Aquí se evidencia que el más bajo es la empatía y el más alto la responsabilidad. Al establecerse que el nivel de calidad de servicio es bajo, nos indica que las dimensiones consideradas para la evaluación pocas veces son aplicadas con efectividad para el desarrollo de un ambiente favorable para los usuarios que buscan a diario una atención.

Tabla l. Calidad de servicio

\begin{tabular}{lcc}
\hline \multicolumn{1}{c}{ DIMENSIONES } & Media & $\begin{array}{c}\text { Desviación } \\
\text { estándar }\end{array}$ \\
\hline \hline TANGIBILIDAD & 2,22 &, 64 \\
SEGURIDAD & 2,21 &, 65 \\
RESPONSABILIDAD & 2,23 &, 67 \\
EMPATÍA & 2,19 &, 69 \\
\hline \hline
\end{tabular}

Fuente: Cuestionario aplicado a los usuarios del área de atención al asegurado del Hospital José Cayetano Heredia.

En la tabla 2 se evidencia que el resumen descriptivo del nivel de satisfacción de los usuarios en el área indica un promedio de 2.21 puntos, considerándose como bajo. A la vez se denota los promedios en cada uno de las dimensiones que la componen. En el caso de la receptibilidad el nivel de satisfacción es bajo con 2.25 en promedio, al evaluarse que el personal del área casi no brinda el servicio con la prontitud que los usuarios esperan, al presentarse un problema no hay un sincero interés por solucionarlo, así como también si el usuario requiere algún servicio o bien adicional, el personal no muestra interés por acudir a 
REVISTA DE LA UNIVERSIDAD DEL ZULIA. 3época. Año $11 \mathrm{~N}^{\circ}$ 31, 2020

su requerimiento. Para la dimensión de la credibilidad el nivel de calidad es baja con un 2.21 en promedio, reflejado en la falta de comunicación, escasa información y conocimiento con los usuarios cuando se presenta algún problema en el servicio, así mismo cuando no se comunican los horarios exactos de los servicios. Con relación a la dimensión competencia se muestra que en promedio es 2.19 puntos, mostrándose un nivel de satisfacción baja. Los promedios ligeramente muestran puntos entre 2.19 y 2.25, evidenciándose el más bajo la competencia y el más alto la receptividad.

Tabla 2. Nivel de satisfacción del usuario

\begin{tabular}{lcc}
\hline \hline \multicolumn{1}{c}{ DIMENSIONES } & Media & Desviación estándar \\
\hline \hline RECEPTIVIDAD & 2,25 &, 61 \\
CREDIBILIDAD & 2,21 &, 69 \\
COMPETENCIA & 2,19 &, 71 \\
\hline & & \\
SATISFACCIÓN DEL & 2,21 &, 65 \\
USUARIO & & \\
\hline
\end{tabular}

Fuente: Cuestionario aplicado a los usuarios del área de atención al asegurado del Hospital José Cayetano Heredia.

En la tabla 3 se demuestra que para hallar las correlaciones entre la calidad de servicio y la satisfacción de los usuarios y cada una de las dimensiones evaluadas, se empleó la correlación no paramétrica de Spearman a un nivel de significancia del $0.05 \%$, donde se halló que la calidad de servicio evidencia una correlación significativa, evidenciando que si existe una buena calidad de servicio para los usuarios, lo que determina que tanto el recurso humano, como la tangibilidad influyen en demasía en la calidad de servicio que ofrece el área de atención al asegurado del Hospital José Cayetano Heredia, de tal manera también en la satisfacción del usuario, coincide con los hallazgos de Civera (2008), que considera que el recurso humano tiene gran influencia en la calidad, así mismo en el Perú no es lo contrario, ya 
REVISTA DE LA UNIVERSIDAD DEL ZULIA. 3época. Año $11 \mathrm{~N}^{\circ}$ 31, 2020

que para lograr calidad en el servicio, el recurso humano debe estar bien entrenado, estos resultados se evidencian en la investigación de (Montalvo et al, 2020), al demostrar que si existe relación significativa entre la calidad de servicio con la satisfacción de los clientes.

Por otro lado, las dimensiones evaluadas para la calidad de servicio como la tangibilidad, seguridad, responsabilidad y empatía evidenciaron niveles bajos en un 47,1\%, $50 \%, 41,4 \%$ y $51.4 \%$ consecutivamente. Estos resultados se vieron reflejados en la investigación de Civera (2008), donde los principales elementos o factores que mayor influencia tienen en la formación de dimensiones de calidad percibida por los clientes externos, son la empatía, condiciones de ética, responsabilidad, sociales y comportamientos estrictamente científicos y sanitarios es decir la profesionalidad del personal.

Con respecto a los servicios intangibles, enfatizamos al servicio percibido por el cliente, como la seguridad, responsabilidad, empatía, receptividad, credibilidad y competencia, de acuerdo a Landa (2015), afirma que en general, las prestadoras de salud, deben hacer sentir a los pacientes que son importantes y especiales, y que la prestadora se preocupa por ellos, por su intereses y necesidades, como una orientación estratégica centrada en mantener y fortalecer los vínculos con los usuarios que permita mejorar la calidad de vida de la población. Sin embargo, en el área de atención al asegurado se reflejó un nivel de calidad baja en todas las dimensiones intangibles, debido a la falta de interés en capacitar al personal.

\section{Conclusiones}

- La calidad de servicio muestra una correlación significativa positiva alta con la satisfacción del usuario al mostrar un resultado de 0.88 , evidenciando que si existe una buena calidad de servicio para los usuarios, éstos a la vez obtendrán la satisfacción completa cuando reciban los servicios que se prestan en el área de atención al asegurado del Hospital José Cayetano Heredia -Piura.

- Todo establecimiento de salud debe contar con personal altamente capacitado para abastecer la demanda del servicio de una comunidad, así mismo, debe estar implementado, con equipos de última generación, medicina, tecnología, recurso humano altamente capacitado, con infraestructura adecuada, etc. Cabe resaltar que es un derecho fundamental de todo ser humano recibir un buen servicio en el sector salud que brinde seguridad y confianza al usuario. 
REVISTA DE LA UNIVERSIDAD DEL ZULIA. $3^{a}$ época. Año 11 N 31, 2020

Teolinda Chuquicusma Tocto et al // Calidad de servicio y nivel de satisfacción... 39-51

DOI: http://dx.doi.org/10.46925//rdluz.31.04

Tabla 3. Relación entre la calidad de servicio y satisfacción del usuario

\begin{tabular}{|c|c|c|c|c|c|}
\hline \multicolumn{2}{|c|}{ DIMENSIONES } & $\begin{array}{l}\text { RECEPTIVI- } \\
\text { DAD }\end{array}$ & $\begin{array}{l}\text { CREDIBILI- } \\
\text { DAD }\end{array}$ & $\begin{array}{l}\text { COMPE- } \\
\text { TENCIA }\end{array}$ & $\begin{array}{l}\text { SATISFAC- } \\
\text { CIÓN }\end{array}$ \\
\hline \multirow[t]{2}{*}{ TANGIBILIDAD } & $\begin{array}{l}\text { Coeficiente de } \\
\text { correlación }\end{array}$ &, $722^{* *}$ &, $727^{* *}$ &, $739^{* *}$ &, $745^{* *}$ \\
\hline & Sig. (bilateral) &, 000 &, 000 &, 000 &, 000 \\
\hline \multirow[t]{2}{*}{ SEGURIDAD } & $\begin{array}{l}\text { Coeficiente de } \\
\text { correlación }\end{array}$ &, $759^{* *}$ &, $783^{* *}$ &, $751^{* *}$ &, $780^{* *}$ \\
\hline & Sig. (bilateral) &, 000 &, 000 &, 000 &, 000 \\
\hline \multirow[t]{2}{*}{$\begin{array}{c}\text { RESPONSABILI- } \\
\text { DAD }\end{array}$} & $\begin{array}{l}\text { Coeficiente de } \\
\text { correlación }\end{array}$ &, $823^{* *}$ &, $880^{* *}$ &, $855^{* *}$ & $897^{* *}$ \\
\hline & Sig. (bilateral) & ,000 &, 000 &, 000 & ,000 \\
\hline \multirow[t]{2}{*}{ EMPATÍA } & $\begin{array}{l}\text { Coeficiente de } \\
\text { correlación }\end{array}$ &, $821^{* *}$ &, $839^{* *}$ &, $844^{* *}$ &, $870^{* *}$ \\
\hline & Sig. (bilateral) &, 000 &, 000 &, 000 &, 000 \\
\hline \multirow[t]{2}{*}{$\begin{array}{l}\text { CALIDAD } \\
\text { SERVICIO }\end{array}$} & $\begin{array}{l}\text { Coeficiente de } \\
\text { correlación }\end{array}$ &, $822^{* *}$ &, $860^{* *}$ &, $857^{* *}$ &, $888^{* *}$ \\
\hline & Sig. (bilateral) &, 000 &, 000 &, 000 &, 000 \\
\hline
\end{tabular}

Fuente: Cuestionario aplicado a los usuarios del área de atención al asegurado del Hospital José Cayetano Heredia.

** La correlación es significativa al nivel 0,05 (bilateral)

\section{Referencias}

Canzio, C. (2019). Relación entre calidad de servicio y satisfacción del usuario en emergencia de hospitales públicos de Lima Este, 2018. Tesis. Universidad San Ignacio de Loyola, Lima, Perú.

Cadena, J., Cruz, V., León, J. y Cadena, G. (2019), Gestión de procesos y satisfacción del usuario en centros de salud de los cantones Quito y Rumiñahui (Ecuador). Revista Espacios vol. $40 \quad$ (37), 4 pp. 23. Recuperado de: https://www.revistaespacios.com/a19v40n37/19403723.html

Castellano, S., Peña, O. y Suárez, D. (2019). Calidad de servicio en centros materno-pediátricos del estado Zulia en Venezuela. Revista Venezolana de Gerencia, Vol. 24, Núm. 86, pp. 395-415. Recuperado de: http://produccioncientificaluz.org/index.php/rvg/article/view/23770

Casaño, B. (2017). Calidad de atención y satisfacción del usuario en el servicio de emergencia del Hospital Grau, 2017. (Tesis de Maestría) Universidad Cesar Vallejo.

Civera, M. (2008). Análisis de la relación entre calidad y satisfacción en el ámbito Hospitalario en función del Modelo de Gestión establecido. Universidad Jume. Facultad de Ciencias Jurídicas y Económicas. Departamento de administración de empresas y Marketing. 
REVISTA DE LA UNIVERSIDAD DEL ZULIA. 3época. Año $11 \mathrm{~N}^{\circ}$ 31, 2020

Teolinda Chuquicusma Tocto et al // Calidad de servicio y nivel de satisfacción... 39-51

DOI: http://dx.doi.org/10.46925//rdluz.31.04

Cobo, E., Estepa, K., Herrera, C., y Linares, P. (2018). Percepción de los usuarios frente a la calidad del servicio de salud en consulta externa en una Institución Prestadora de Servicios de Salud. Revista Investigación en Salud Universidad de Boyacá, 5(2), 277-294. Recuperado de: https://doi.org/10.24267/23897325.321

Deming, W.E. (1989). Calidad, productividad y competitividad. La salida de la crisis. Madrid: Díaz de Santos.

Fariño, J., Cercado, A., Vera, E., VALLE, J. y Ocaña, A. (2018). Satisfacción de los usuarios y la calidad de atención que se brinda en las unidades operativas de atención primaria de salud. Revista Espacios vol. 39 (32), pp. 22.

Fernández, D., Revilla, M., Kolevic, L., Cabrejos, I., Muchaypiña, I., Sayas, I., Chávez, L. y Mamani, V. (2019). Clima organizacional y satisfacción del usuario externo en los servicios de hospitalización del Instituto Nacional de Salud del Niño, 2017. Anales de la Facultad de Medicina, vol. 80(2), pp. 188-192. Recuperado de: https://revistasinvestigacion.unmsm.edu.pe/index.php/anales/article/view/ 15745

Gallardo, A. y Reynaldos, K. (2014). Calidad de servicio: satisfacción usuaria desde la perspectiva de enfermería. Revista Enfermería Global vol.13 (36), pp. 353-363.

Hernández, R., Fernández, C. \&e Baptista, M. (2010). Metodología de la Investigación. México: McGRAW-HILL.

Juran, J. y Gryna, F. (1993). Manual de control de calidad. México: McGraw-Hill Interamericana.

Landa, O. (2015). La calidad de servicio en la satisfacción que obtienen los usuarios de las entidades prestadoras de salud. (Tesis de maestría) Universidad Nacional de Educación "Enrique Guzmán y Valle".

Loli, A., Del Carpio, J., Cuba, E., Vergara, A., Morales, S., Flores, M., y Lamas, L. (2014). La satisfacción y la calidad de servicio en Organizaciones públicas y privadas de Lima Metropolitana. Revista de Investigación en Psicología, 16(1), 171-190. Recuperado de https://revistasinvestigacion.unmsm.edu.pe/ index.php/ psico/article/view/3926

Ley 26842 “Ley General de Salud”. Perú (1997).

Losada, M., Rodríguez, A. (2007). Calidad del servicio de salud: una revisión a la literatura desde la perspectiva del marketing. Cuadernos de Administración: Vol. 20,(34), pp. 237258. Recuperado de: https://revistas.javeriana.edu.co/index.php/ cuadernos_admon/article/view/4052 
REVISTA DE LA UNIVERSIDAD DEL ZULIA. $3^{a}$ época. Año 11 N 31, 2020

Teolinda Chuquicusma Tocto et al // Calidad de servicio y nivel de satisfacción... 39-51

DOI: http://dx.doi.org/10.46925//rdluz.31.04

Menacho, I., Mallqui, V., Ibarguen, F. y Córdova, U. (2020). Calidad de servicio e Imagen corporativa en EsSalud, Perú. Revista Venezolana de Gerencia, vol. 25 (91), pp. 1190-1204. Disponible en: Recuperado de: http:/produccioncientificaluz.org/index.php/rvg/article/view/33198/34857

Montalvo, S. y Estrada, E. y Mamani, H. (2020). Calidad de servicio y satisfacción del usuario en el Hospital Santa Rosa de Puerto Maldonado. Revista Ciencia y Desarrollo. Universidad Alas Peruanas vol. 23 (2), pp. 2l-26. Recuperado de: http://revistas.uap.edu.pe/ojs/index.php/CYD/article/view/2085

Numpaque, A., Buitrago, L. y Pardo, D. (2019). Calidad de la atención en el servicio de cirugía ambulatoria desde la percepción del usuario. Revista de la Facultad de Medicina, vol. 67 (2), pp. 235-239. Recuperado https://revistas.unal.edu.co/index.php/revfacmed/article/view/65978

Ortiz, P. (2016). Nivel de satisfacción del usuario externo sobre la calidad de atención en los servicios de salud en la Micro Red Villa - Chorrillos en el año 2014. (Tesis de Licenciatura) Universidad Ricardo Palma, Lima, Perú.

Pantoja, M. y Salazar, J. (2019). Etapas de la administración: hacia un enfoque sistémico. Revista Escuela de Administración de Negocios, № 87, pp. 139-154. Recuperado de: https:/journal.universidadean.edu.co/index.php/Revista/ article/ view/2412

Parasuraman, A., Zeithaml, V. and Berry, L. L. (1985). A conceptual model of service quality and its implications for future research. Journal of Marketing, 49 (4), 41-50.

Podestá, L. y Maceda, M. (2018). Calidad percibida del servicio y cultura de seguridad en salud en el personal médico del Hospital II EsSalud Vitarte. Lima, 2017. Horiz Med, vol. 18(3), pp. 48-56.

Soto, P., Virú, M., Elorreaga, O., Amaya, E., Mezones, E., Ramírez, R., Tizón, M., Hurtado, Y., Pimentel, P., Cuba, W., y Suarez, V. (2020). Factores asociados a la calidad de la atención en población adulta afiliada a la Seguridad Social: El caso peruano. Revista Del Cuerpo Médico Del HNAAA, 13(1), pp. 14 - $25 . \quad$ Recuperado de: http://cmhnaaa.org.pe/ojs/index.php/rcmhnaaa/article/view/595

Vásquez Gastelumendi; D. V.; Ríos Campos, C. A.; Santamaría Baldera, N.; Gutiérrez Valverde, K. S.; Camacho Delgado, F. M.; Aguirre Zaquinaula, I. R.; Estela Urbina, R. O. (2019). Estrategias organizacionales para fortalecer el clima laboral en la Escuela Profesional de Tecnología Médica - Facultad de Ciencias de la Salud de la Universidad de Chiclayo, Revista de la Universidad del Zulia, $10 \quad$ (28), $112-136$ https://produccioncientificaluz.org/index.php/rluz/article/view/30602

Zeithmal, V. y Bitner J. (2002). Marketing de servicio. 2da Edición. Editorial: Fic Graw-Hill Interamericana. 